\title{
BMJ Open High-risk factors for adverse pregnancy outcomes in systemic lupus erythaematosus: a retrospective study of a Chinese population
}

\author{
Meng Jiang, ${ }^{1,2}$ Yanling Chang, ${ }^{1,2}$ You Wang, ${ }^{1,2}$ Qiong Fu (D) , ${ }^{3,4}$ Sihan Lin,, \\ Jiayue Wu (D) , ${ }^{1,2}$ Wen $\mathrm{Di}^{1,2,5}$
}

To cite: Jiang M, Chang $Y$, Wang Y, et al. High-risk factors for adverse pregnancy outcomes in systemic lupus erythaematosus: a retrospective study of a Chinese population. BMJ Open 2021;11:e049807. doi:10.1136/ bmjopen-2021-049807

- Prepublication history and additional supplemental material for this paper are available online. To view these files, please visit the journal online (http://dx.doi.org/10.1136/ bmjopen-2021-049807).

$\mathrm{MJ}$ and $\mathrm{YC}$ contributed equally.

$\mathrm{MJ}$ and $\mathrm{YC}$ are joint first authors.

Received 04 February 2021 Accepted 20 October 2021

Check for updates

(c) Author(s) (or their employer(s)) 2021. Re-use permitted under CC BY-NC. No commercial re-use. See rights and permissions. Published by BMJ.

For numbered affiliations see end of article.

\section{Correspondence to}

Dr Jiayue Wu;

janet_wu_jiayue@163.com and

DrWen Di;

diwen163@163.com

\section{ABSTRACT}

Objective To clarify high-risk factors for adverse pregnancy outcomes (APOs) in systemic lupus erythaematosus (SLE).

Design A retrospective chart review study.

Setting Data were collected in a tertiary medical centre, Shanghai, China, from November 2010 to December 2018. Participants A total of 513 pregnancies with SLE were retrospectively analysed. Twenty-seven patients who underwent artificial abortions due to personal reasons were excluded.

Primary outcome measures APOs were primary outcomes, including foetal loss, premature birth, small for gestational age (SGA), asphyxia neonatorum, composite foetal APOs and hypertensive disorders of pregnancy (HDP). Multivariable logistic regression and Spearman correlation analysis were performed to determine the risk factors for APOs in SLE.

Results Risk factors for foetal loss included prepregnancy hypertension, hypocomplementaemia-C3, anticardiolipin antibodies-IgM positivity and disease flares during pregnancy. Risk factors for premature birth included disease flares, use of immunosuppressive agents and HDP. Moreover, twin pregnancy, disease flares and HDP were risk factors for SGA, and prepregnancy hypertension was an independent risk factor for asphyxia neonatorum. Independent risk factors for composite foetal APOs included twin pregnancy, prepregnancy hypertension, disease flares during pregnancy, $\mathrm{HDP}$, hypocomplementaemia-C3 and the use of immunosuppressive agents. Risk factors for SLE complicated with HDP included prepregnancy hypertension, renal disorders and thrombocytopaenia. Conversely, the use of aspirin was a protective factor against foetal loss and premature birth. The ds-DNA value had a low diagnostic value for APOs, whereas the extent of complement reduction may predict the incidence of composite foetal APOs and foetal loss. Proteinuria occurring in the first 20 gestational weeks may lead to APOs.

Conclusion Established risk factors for each APO were identified in this study. Indicators with more predictive significance have been screened out from conventional indicators, which may help clinicians predict the pregnancy outcome of patients with SLE more accurately and minimise the incidence of APOS.
Strengths and limitations of this study

- A comprehensive analysis was performed of the most important risk factors for the main maternal and foetal adverse pregnancy outcomes (APOs) caused by placental dysfunction in systemic lupus erythaematosus (SLE) pregnancy with a large sample size.

- The study demonstrated that the ds-DNA value had a low diagnostic value for APOs, whereas the extent of complement decrease, especially $\mathrm{C} 3$, may predict the incidence of composite foetal APOs, especially foetal loss.

- The study contributes to a better counselling of obstetric surveillance in SLE pregnancy.

- As a retrospective study, inherent information bias was present.

\section{INTRODUCTION}

Systemic lupus erythaematosus (SLE) is an autoimmune disease involving multiple organs and autoantibodies. Nearly 90\% of females with SLE are of reproductive age. ${ }^{1}$ Previous epidemiological studies have demonstrated that the prevalence and incidence rates of patients with SLE among Asians are approximately 2-3 times higher than those among Caucasians. China has a higher prevalence of SLE than many other countries, especially among women (estimated to be more than 100 per 100000 persons). Based on an estimated Chinese population of 1.3 billion published in 2009 , the number of lupus patients in China could reach 520000-910000, which would be the largest cluster of cases in the world. ${ }^{2}$ To tolerate the paternal antigens expressed in foetal cells or tissues, the maternal immune system may undergo adaptive changes during pregnancy, which can stimulate the autoimmune response and lead to SLE flares. The flare rate in pregnancy has been reported 
to range from $13 \%$ to $68 \%$, accompanied by irreversible organ damage and adverse pregnancy outcomes (APOs). ${ }^{3}$ Although diagnostic and therapeutic strategies for SLE have greatly improved, SLE in pregnancy is still a high risk factor due to frequent complications, including preeclampsia (PE), small for gestational age (SGA), foetal loss and premature birth. ${ }^{45}$

Prepregnancy counselling and perinatal care are essential for the prevention of APOs in the SLE population. Indeed, potential clinical risk factors and serological predictors of adverse outcomes in SLE pregnancies have been widely studied in recent decades. ${ }^{6-13}$ Nevertheless, there is no consensus regarding predictors for each APO, and most risk factors are presented as categorical variables. Given the different incidences of SLE in various countries and the limitation of methodology consistency, there is a need for a concise and evidencebased list of indicators to estimate SLE pregnancy risk. In addition, it remains unknown whether the extent of the abnormality of disease activity indexes for SLE, such as ds-DNA, complement and proteinuria, can accurately predict pregnancy outcomes. Furthermore, there are few studies involving large samples. Ren Ji Hospital has treated a leading number of SLE pregnancies in China, which provided our study with a rare large single-centre sample. Here, we evaluated 513 pregnant women and analysed high-risk factors for adverse SLE maternal and foetal outcomes to strengthen management and improve SLE pregnancy outcomes.

\section{METHODS}

\section{Patient population}

This was a retrospective study performed at Ren Ji Hospital, Shanghai Jiao Tong University School of Medicine, Shanghai, China. The medical records of all pregnant patients with SLE (meeting $\geq 4$ of the revised American College of Rheumatology criteria ${ }^{14}$ ) between November 2010 and December 2018 were reviewed. The total number of deliveries in our hospital during the study period reached 24 859, with SLE pregnancies accounted for $2.2 \%$. Twenty-seven patients who underwent artificial abortions due to personal reasons rather than therapeutic reasons were excluded. Rheumatologists diagnosed and obstetricians jointly managed SLE pregnant women.

\section{Variables of interest}

Clinical and laboratory information was recorded from the first antenatal care records (16-20 gestational weeks). Baseline maternal information included age, obstetric history, duration of SLE, previous manifestations of SLE (including renal disorders, mucocutaneous disorders, haematological disorders, neurological disorders, arthritis and serositis) and medication use. Comorbidities included prepregnancy hypertension and diabetes. Laboratory data collected included 24-hour urinary protein, antinuclear antibodies, complement 3 (C3), complement 4 (C4), ds-DNA and antiphospholipid antibody (aPL) results. aPL included $\mathrm{IgG} / \mathrm{IgM}$ anticardiolipin antibodies (aCLs) and anti-2-glycoprotein I antibodies (anti- $\beta 2 \mathrm{GPI}$ ); only titres of aCLs, $\beta 2 \mathrm{GPI}$ IgG, IgM $\geq 40$ GPL or MPL units were considered positive. All laboratory tests were performed using standardised methods. Each pregnancy was recorded as a separate observation. Pregnancy outcomes were also evaluated, including delivery mode, foetal survival, Apgar score and foetal birth weight.

\section{Patient and public involvement}

Patients and the public were not involved in the design and conception of the study and there are no plans to disseminate the results to patients.

\section{Definitions}

Foetal APOs included one or more of the following: (1) foetal loss-spontaneous abortion (referring to termination before 28 weeks of pregnancy with foetal weight less than $1000 \mathrm{~g}$ ), therapeutic abortion (iatrogenic abortion caused by a lupus flare or obstetric complications threatening the life of the mother), stillbirth (any baby born without signs of life at $\geq 28$ completed weeks of gestation), and neonatal death (death of a liveborn baby within 28 days after birth $)^{15}$; (2) premature birth-delivery prior to 37 weeks of gestation ${ }^{16}$; (3) SGA-birth weight below the 10th percentile according to gestational week at delivery and foetal $\operatorname{sex}^{17}$; and (4) asphyxia neonatorum-Apgar score of $<7$ at 1 and/or 5 min after birth. ${ }^{18}$ Composite foetal APOs were defined as the occurrence of any adverse outcomes, including foetal loss, premature birth, SGA and asphyxia neonatorum.

Hypertensive disorders of pregnancy (HDPs) were categorised into three types in this study. (1) Gestational hypertension (GH): new-onset blood pressure $\geq 140 / 90 \mathrm{~mm} \mathrm{Hg}$ without proteinuria after 20 weeks of gestation. (2) PE: the first incidence of systolic blood pressure (SBP) $\geq 140 \mathrm{~mm}$ $\mathrm{Hg}$ and/or diastolic blood pressure (DBP) $\geq 90 \mathrm{~mm} \mathrm{Hg}$ after 20 weeks of gestation plus one of the following criteria, protein loss of $300 \mathrm{mg}$ or more in a 24-hour urine specimen or maternal organic dysfunction, such as loss of renal function, hepatic dysfunction, neurological complications (altered mental state, blindness, scotomas, visual blurring), haematological complications (thrombocytopaenia, haemolysis) or intrauterine growth restriction; PE can also overlap with other hypertensive states, such as prepregnancy hypertension preceding pregnancy or identified before 20 weeks. (3) Eclampsia: new-onset generalised seizures in a woman with PE. ${ }^{19}$

A disease flare during pregnancy was defined as a new or worsened presence of arthritis, malar rash, vasculitis, oral or nasal ulcers, serositis, neurological manifestations, haematological disorders, fever attributable to SLE, the addition of immunosuppressive medications or hydroxychloroquine, or an increase in prednisone $\geq 0.5 \mathrm{mg} / \mathrm{kg}$ / day. Additionally, new-onset SLE during pregnancy was included. ${ }^{20}$ 


\section{Statistical analyses}

Continuous variables were analysed using analysis of variance tests when the distributions were normal or KruskalWallis $\mathrm{H}$ tests when the distributions were not normal, and the results are presented as the mean $\pm \mathrm{SD}$ or as the frequency. Categorical variables were analysed using $\chi^{2}$ or Fisher's exact probability tests as appropriate. Multivariable and stepwise regression $(\mathrm{p}<0.05$ for forward steps and $\mathrm{p}<0.10$ for backward steps) was performed by selecting variables with a $p$ value $<0.05$ in the univariate analysis. For categorical variables, univariate ORs and corresponding 95\% CIs were computed. Spearman tests were employed to determine correlations between variables. The area under the receiver operating characteristic (ROC) curve (AUC) was used to assess discrimination of continuous variables with a $\mathrm{p}$ value $<0.05$ in the Spearman test and to obtain the critical cut-off value. All tests were two-tailed, and $\mathrm{p}<0.05$ was considered statistically significant. All analyses were performed using SPSS V.25.0.

\section{RESULTS}

\section{Population characteristics}

A total of 513 pregnancies in 484 patients with SLE were recorded at Ren Ji Hospital, Shanghai Jiao Tong University School of Medicine, between November 2010 and December 2018. Of these patients, $456(94.2 \%)$ had 1 pregnancy within the study period, 27 (5.6\%) had 2 pregnancies and $1(0.2 \%)$ had three pregnancies. Through retrieval by case diagnosis, 41 cases of antiphospholipid antibody syndrome were identified among patients with SLE. The mean age at conception was $29.7 \pm 4.0$ years (range, 20-40 years). The average duration of SLE before pregnancy was $6.6 \pm 4.3$ years (range, $1-18$ years). There were 238 cases $(46.4 \%)$ of primipara, 505 cases $(98.4 \%)$ of singleton pregnancy and 8 cases $(1.6 \%)$ of twin pregnancy. Twenty-one patients $(4.1 \%)$ had prepregnancy hypertension. Almost $96 \%$ of the patients (495 cases) were in the SLE remission stage for more than 6 months prior to conception. Eighty-two of the patients $(16 \%)$ had a disease flare before 20 weeks of gestation. A total of 501 patients $(97.7 \%)$ used prednisone, $405(78.9 \%)$ took hydroxychloroquine and $45(8.8 \%)$ received immunosuppressive medications (such as azathioprine, tacrolimus and cyclosporine A). Of the patients, 398 (77.6\%) used aspirin, and 138 (26.9\%) received low-molecularweight heparin.

\section{Foetal outcomes}

A total of 444 pregnancies $(86.5 \%)$ resulted in live births. The average gestation days for the live births were $260.10 \pm 15.06$ days (range, 201-282 days), and the average foetal weight was $2797.96 \pm 563.951 \mathrm{~g}$ (range, 940-4370 g). In total, $128(24.9 \%)$ premature births were recorded, and there was no significant difference in the premature birth rate between twins and singletons $\left(\chi^{2}=115.28\right.$, $\mathrm{p}=0.09$ ).
There were 11 cases $(2.1 \%)$ with an Apgar score $<7$ at $1 \mathrm{~min}$ after birth. Only one newborn had Apgar scores $<7$ at 5 and $10 \mathrm{~min}$ after rescue and ultimately died due to oedema. In all cases of asphyxia neonatorum, there was no evidence of cardiac malformations based on B-ultrasound during pregnancy. The overall foetal loss rate was $13.6 \%$ (70 cases), and the SGA rate was $23.4 \%$ (120 cases). There were 236 cases $(46.0 \%)$ with composite foetal APOs.

\section{Maternal outcomes}

In this study, 145 patients (28.3\%) experienced disease flares during pregnancy. Among 513 pregnancies, 90 patients (17.5\%) eventually developed HDP, 16 patients (3.1\%) had GH, 74 patients (14.4\%) had PE and 2 developed eclampsia $(0.4 \%)$. All patients with disease flares and HDPs received timely diagnosis and treatment. One maternal death occurred in a patient with lupus that remained active without evaluation by the rheumatologist or obstetrician after conception. This patient was 30 years old and dramatically deteriorated with pulmonary haemorrhage, and multiple organ failure developed 15 days after iatrogenic abortion.

\section{Predictors of adverse foetal and maternal outcomes}

Table 1 provides a comparison of clinical events as well as laboratory parameters in patients with or without composite foetal APOs. Multivariable analysis revealed that multiple pregnancies, prepregnancy hypertension, disease flares during pregnancy, HDP, hypocomplementaemia-C3 and the use of immunosuppressive agents were independent predictors of composite foetal APOs (table 2).

Univariate analysis of foetal APOs is shown in online supplemental table 1. Multivariable analysis revealed that prepregnancy hypertension, hypocomplementaemia-C3, aCL-IgM positivity and disease flares during pregnancy were risk factors for foetal loss. Disease flares during pregnancy, HDPs and the use of immunosuppressive agents were responsible for premature birth, and multiple pregnancies, disease flares during pregnancy and HDPs were independent predictors of SGA. Moreover, the occurrence of asphyxia neonatorum correlated significantly only with prepregnancy hypertension (table 3).

The maternal characteristics significantly associated with HDPs in the univariate analysis are shown in table 4. In the multivariable analysis, prepregnancy hypertension $(\mathrm{OR}=9.03)$, renal disorders $(\mathrm{OR}=2.71)$ and thrombocytopaenia $(\mathrm{OR}=3.24)$ were independent risk factors for HDP (online supplemental table 2).

The influence of anti-dsDNA, complements and proteinuria on APOs in SLE pregnancies

The results showed that anti-dsDNA correlated slightly positively with the occurrence of foetal loss $(\rho=0.147$, $\mathrm{p}<0.01)$. The value of ds-DNA was converted into a categorical variable according to the critical cut-off value obtained with the ROC curve. Ds-DNA $\geq 14.41 \mathrm{IU} /$ 
Table 1 Maternal characteristics according to composite foetal adverse pregnancy outcomes (APOs)

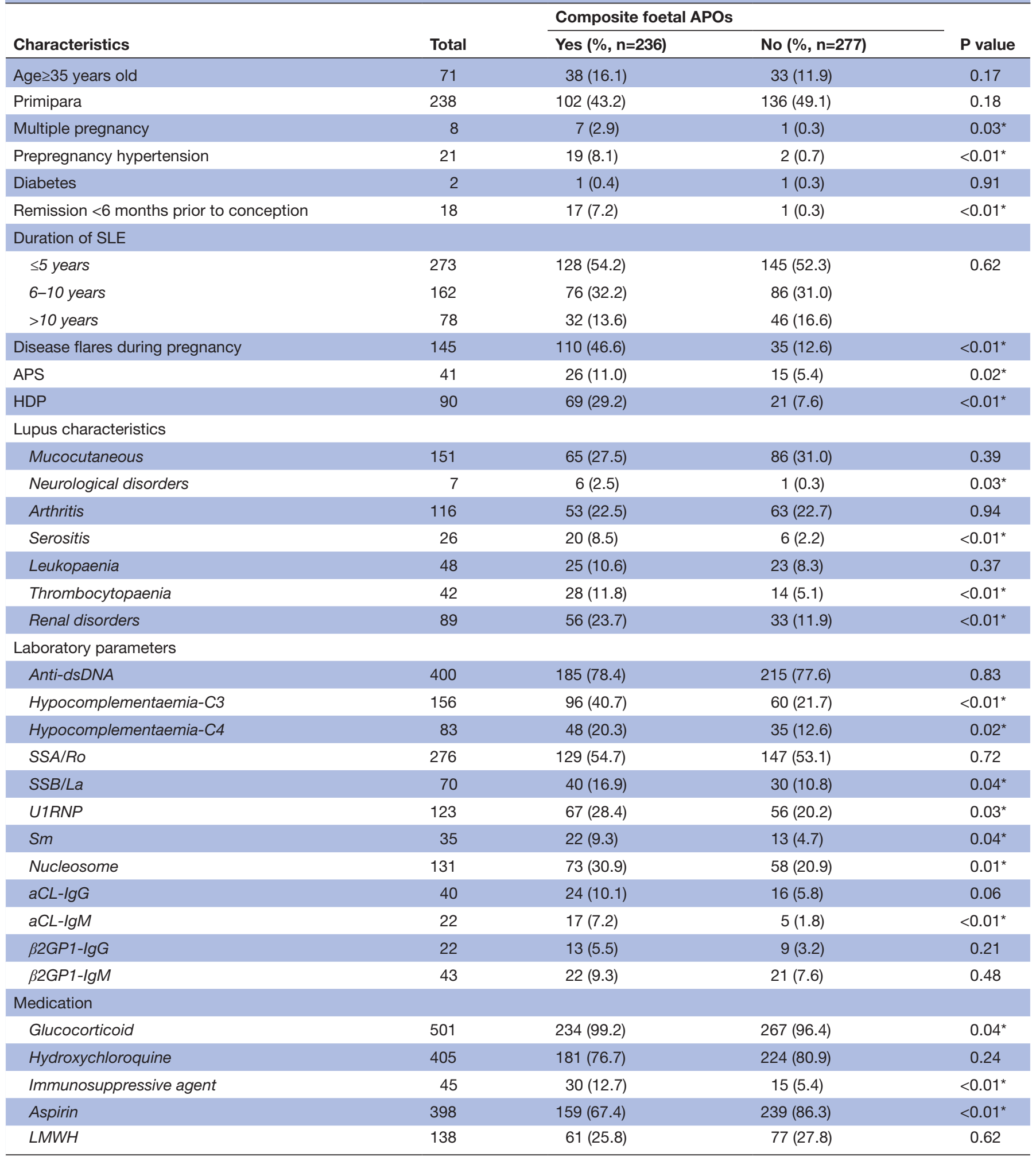

${ }^{*} \mathrm{P}<0.05$.

APS, antiphospholipid antibody syndrome; HDP, hypertensive disorders of pregnancy; LMWH, low-molecular-weight heparin.

$\mathrm{mL} \quad(\mathrm{AUC}=0.624, \quad \mathrm{YI}=0.201$, sensitivity $=0.686$, specificity $=0.515$ ) was found to be a risk factor for foetal loss among pregnant women with SLE. An AUC of less than 0.7 indicates a low diagnostic value of the optimal cut-off value. There was no significant correlation between antidsDNA and other APOs $(p>0.05)$.

To clarify the impact of the degree of decrease in C3 on composite foetal APOs, foetal loss and HDP, the 
Table 2 Predictors of composite foetal adverse pregnancy outcomes (APOs): results of multivariable analysis

\begin{tabular}{|c|c|c|c|c|}
\hline Characteristics & B & P value & OR & $95 \%$ Cls \\
\hline Multiple pregnancy & 2.368 & 0.03 & 10.67 & 1.22 to 93.31 \\
\hline Prepregnancy hypertension & 2.143 & $<0.01$ & 8.52 & 1.81 to 40.21 \\
\hline Disease flares during pregnancy & 1.395 & $<0.01$ & 4.03 & 2.51 to 6.50 \\
\hline HDP & 1.114 & $<0.01$ & 3.05 & 1.69 to 5.47 \\
\hline Hypocomplementaemia-C3 & 0.543 & 0.02 & 1.72 & 1.11 to 2.67 \\
\hline Use of immunosuppressive agent & 0.856 & 0.02 & 2.35 & 1.15 to 4.82 \\
\hline
\end{tabular}

${ }^{*} \mathrm{P}<0.05$.

HDP, hypertensive disorders of pregnancy.

values of C3 were set according to the interval of every $0.1 \mathrm{~g} / \mathrm{L}$ decrease below the lower normal limit. Following the same method, the values of $\mathrm{C} 4$ were set according to the interval of every $0.01 \mathrm{~g} / \mathrm{L}$ decrease below the lower normal limit (online supplemental tables 3 and 4). In addition to HDPs, we found that in both $\mathrm{C} 3$ and $\mathrm{C} 4$, the incidences of composite foetal APOs and foetal loss in any interval below the lower normal limit increased with the decrease in complement (figure 1).

A total of 140 patients $(27.3 \%)$ had proteinuria before 20 weeks of gestation. The diagnostic criterion for a renal disorder in SLE is 24-hour urinary protein of $>0.5 \mathrm{~g}$, and proteinuria during pregnancy is defined as 24-hour urinary protein of $\geq 0.3 \mathrm{~g}^{21}$. Therefore, all cases were divided into four groups regardless of the diagnosis: $\mathrm{N}$ group ( $\mathrm{n}=373$, without proteinuria), $\mathrm{P} 1$ group $(\mathrm{n}=60$, $0.3 \mathrm{~g} \leq 24$ hour urinary protein $\leq 0.5 \mathrm{~g})$, P2 group $(\mathrm{n}=46$,
$0.5 \mathrm{~g}<24$ hour urinary protein $\leq 1 \mathrm{~g}$ ), and P3 group ( $\mathrm{n}=34$, 24-hour urinary protein $>1 \mathrm{~g}$ ).

As shown in table 5 , foetal birth weights and the duration of pregnancy were highest in the N-group and lowest in the P3 group, both of which showed significant differences from each other group. Overall, the incidences of most APOs were lowest in the $\mathrm{N}$ group. The highest incidences of composite foetal APOs, SGA, HDP and premature birth were detected in the $\mathrm{P} 3$ group compared with the other three groups $(\mathrm{p}<0.05)$. Overall, foetal loss rates were similar in the P1, P2 and P3 groups and were higher than those in the $\mathrm{N}$ group $(\mathrm{p}<0.05)$, and premature birth rates differed significantly between each group, except for the N and P1 groups. There were no significant differences in the incidence of asphyxia neonatorum among the four groups.

\begin{tabular}{|c|c|c|c|c|}
\hline Characteristics & B & $P$ value & OR & $95 \% \mathrm{Cl}$ \\
\hline \multicolumn{5}{|l|}{ Foetal loss } \\
\hline Disease flares during pregnancy & 1.054 & $<0.01$ & 2.87 & 1.45 to 5.69 \\
\hline Hypocomplementaemia-C3 & 1.552 & $<0.01$ & 4.72 & 2.47 to 9.02 \\
\hline Use of aspirin & -1.94 & $<0.01$ & 0.14 & 0.07 to 0.29 \\
\hline \multicolumn{5}{|l|}{ Premature birth } \\
\hline Disease flares during pregnancy & 0.872 & $<0.01$ & 2.39 & 1.48 to 3.84 \\
\hline$H D P$ & 0.585 & 0.02 & 1.79 & 1.07 to 3.00 \\
\hline Multiple pregnancy & 2.085 & $<0.01$ & 8.04 & 1.81 to 35.71 \\
\hline Disease flare during pregnancy & 0.612 & 0.01 & 1.84 & 1.15 to 2.95 \\
\hline$H D P$ & 0.914 & $<0.01$ & 2.49 & 1.49 to 4.15 \\
\hline \multicolumn{5}{|l|}{ Asphyxia neonatorum } \\
\hline Prepregnancy hypertension & 0.914 & $<0.01$ & 2.49 & 1.49 to 0.91 \\
\hline
\end{tabular}

HDP, hypertensive disorders of pregnancy; SGA, small for gestational age. 
Table 4 Maternal characteristics according to hypertensive disorder of pregnancy (HDP)

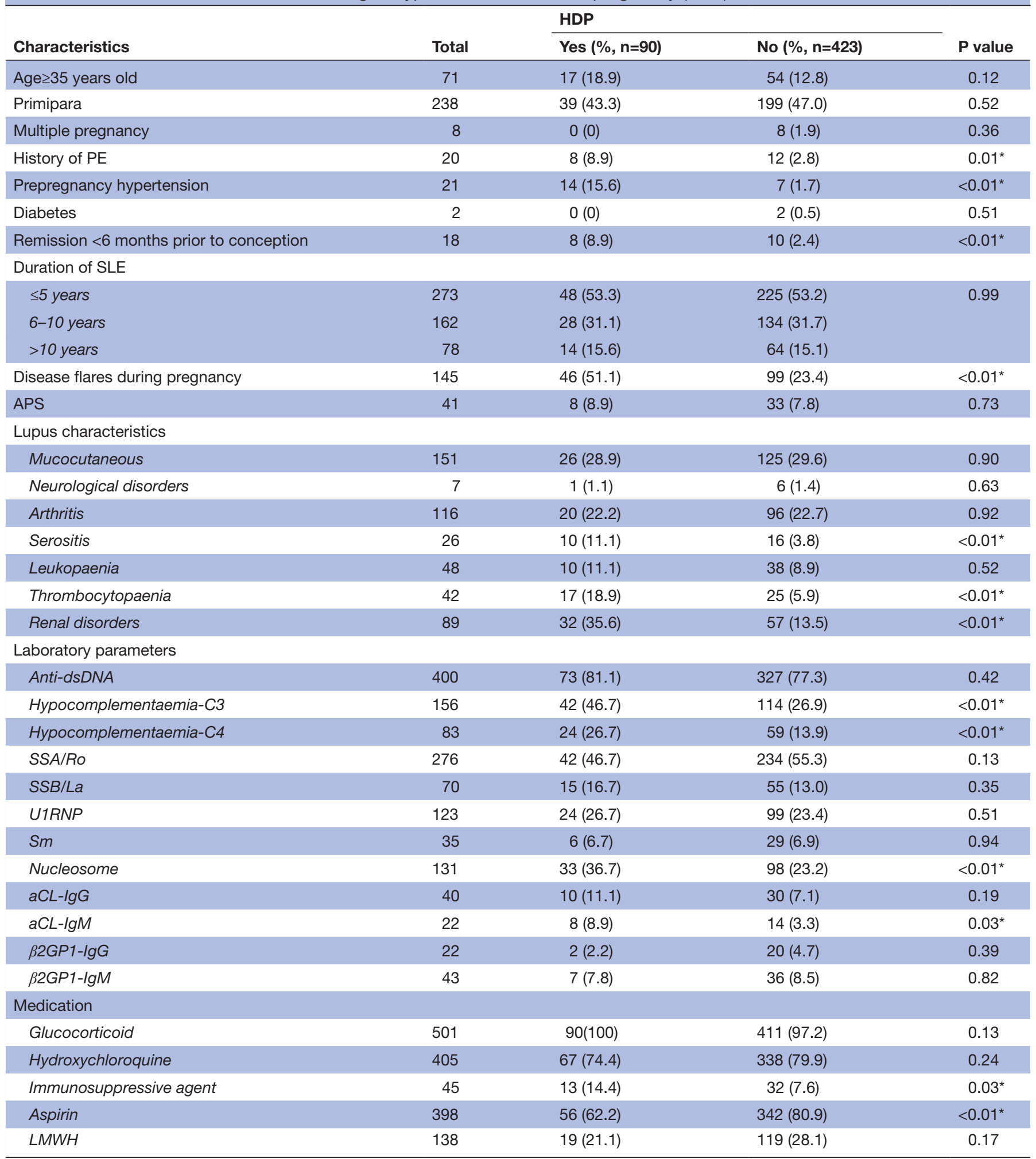

${ }^{*} \mathrm{P}<0.05$.

APS, antiphospholipid antibody syndrome; HDP, hypertensive disorders of pregnancy; LMWH, low-molecular-weight heparin; PE, preeclampsia.

\section{DISCUSSION}

Our study presents a comprehensive analysis of the most important risk factors for each maternal and foetal APO in SLE pregnancy with a large sample size. We found that prepregnancy hypertension, HDP and flares during pregnancy were key risk factors for most APOs. The ds-DNA value had a low diagnostic value for APOs, whereas the extent of complement decrease, especially 

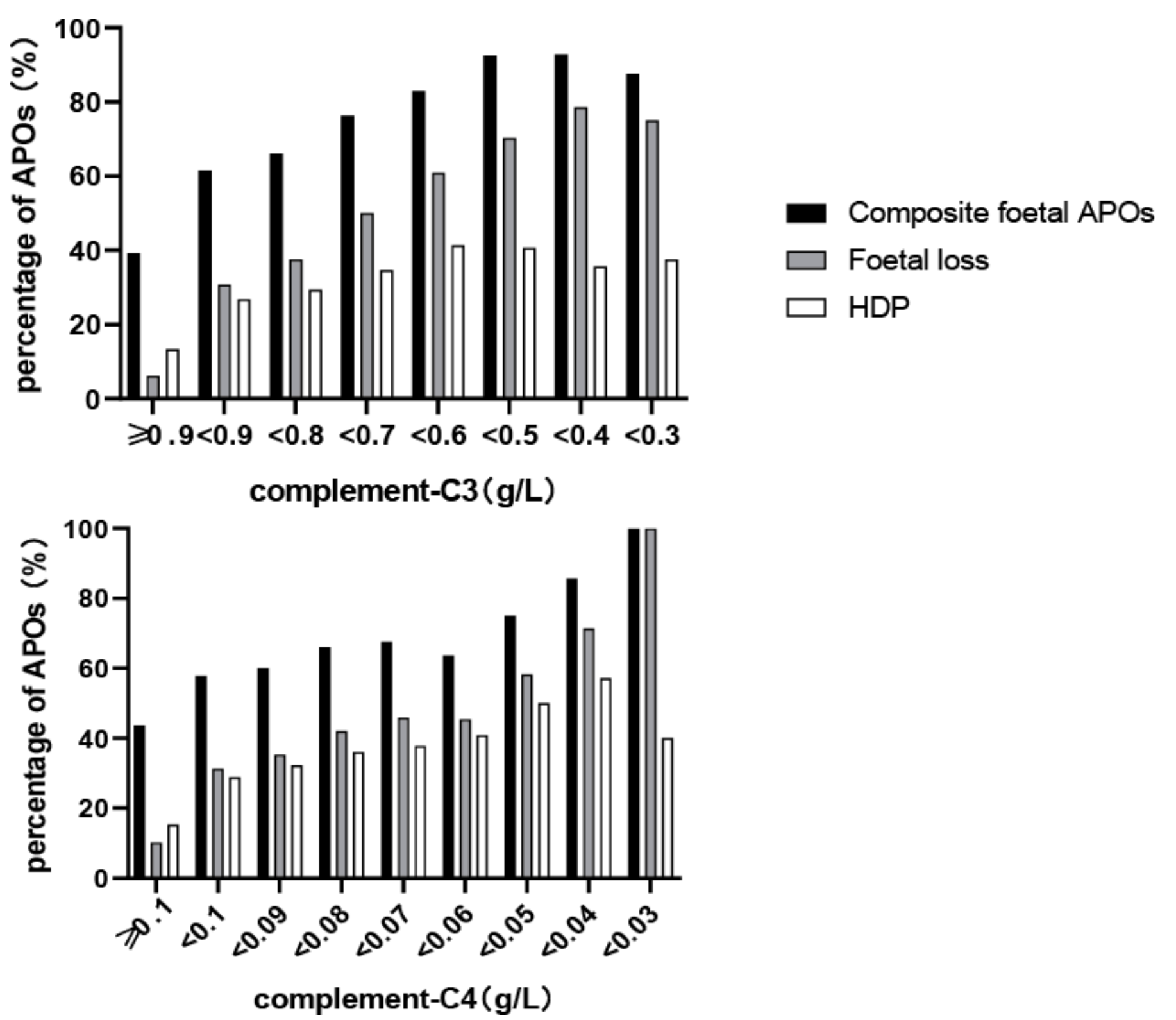

Figure 1 The incidences of adverse pregnancy outcomes (APOs) associated with the different intervals of complement C3 and C4. HDP, hypertensive disorders of pregnancy.

C3, may predict the incidence of composite foetal APOs, especially foetal loss. Proteinuria occurring in the first 20 gestational weeks may lead to APOs.

Patients with SLE have a higher incidence of APOs than the general population, including foetal loss, premature birth, SGA and HDP. ${ }^{22-27}$ Overall, independent risk factors for composite foetal APOs included multiple pregnancies, prepregnancy hypertension, disease flares during pregnancy, HDP, hypocomplementaemia-C3 and the use of immunosuppressive agents, similar to the conclusions of other studies. ${ }^{1222} 28$ Predictors for each outcome are also proposed in this study. The main cause of foetal loss is generally recognised as aPL positivity. ${ }^{29} 30$ Our results showed that aCL-IgM positivity has a greater impact on foetal loss than aCL-IgG or $\beta 2 \mathrm{GPI}$ positivity. In addition, hypocomplementemia-C3, prepregnancy hypertension, and disease flares during pregnancy were independent risk factors for foetal loss, consistent with previous findings. 91524

The main predictors of preterm birth and SGA in previous studies were lupus activity during pregnancy and HDP, ${ }^{22}$ 31-34 which was also confirmed in our study. In addition, immunosuppressant use and disease flares were jointly found to be independent risk factors for preterm birth in the present study, indicating that they may be caused by lupus flares rather than by adverse drug events. ${ }^{35}$ We still felt that immunosuppressant use should be continued in patients who benefit from therapy. Data regarding foetal complications during therapy are scarce, but no evidence of teratogenesis has emerged. In addition, many studies have ruled out the effect of multiple pregnancies on SLE pregnancy. However, the dual factors of a twin pregnancy and an abnormal placenta induced by the disease may aggravate the risk of SGA in patients 
Table 5 Pregnancy outcomes of systemic lupus erythaematosus (SLE) pregnancies with or without proteinuria

\begin{tabular}{|c|c|c|c|c|c|}
\hline Characteristics & $\begin{array}{l}\text { N-group } \\
(n=373, \%)\end{array}$ & $\begin{array}{l}\text { P1-group } \\
(\mathrm{n}=60, \%)\end{array}$ & $\begin{array}{l}\text { P2-group } \\
(n=46, \%)\end{array}$ & $\begin{array}{l}\text { P3-group } \\
(\mathrm{n}=34, \%)\end{array}$ & $P$ value \\
\hline Live birth & $348(93.3)^{*}$ & 44 (73.3) & 32 (69.5) & $20(58.8)$ & $<0.01$ \\
\hline $\begin{array}{l}\text { Foetal birth weight } \\
(g, \text { mean } \pm S D)\end{array}$ & $2887.93 \pm 495.54^{*}$ & $2451.76 \pm 986.67 \dagger$ & $2392.92 \pm 883.68 \dagger$ & $1911.40 \pm 935.857^{*}$ & $<0.01$ \\
\hline $\begin{array}{l}\text { Duration of pregnancy } \\
\text { (days, mean } \pm S D \text { ) }\end{array}$ & $254.62 \pm 34.56^{\star}$ & $226.05 \pm 58.64 \dagger$ & $221.87 \pm 52.84 \dagger$ & $209.18 \pm 53.35^{\star}$ & $<0.01$ \\
\hline \multicolumn{6}{|l|}{ APOs } \\
\hline Composite foetal APOs & $134(35.9)^{\star}$ & $40(66.7) \dagger$ & $33(71.7) \dagger$ & $29(85.3)^{\star}$ & $<0.01$ \\
\hline Foetal loss & $26(6.9)^{\star}$ & $16(26.7)$ & $14(30.4)$ & $14(41.2)$ & $<0.01$ \\
\hline Premature birth & $83(83 / 348,23.8) \ddagger$ & $14(14 / 44,31.8) \ddagger$ & $16(16 / 32,50.0)^{\star}$ & $15(15 / 20,75.0)^{*}$ & $<0.01$ \\
\hline$S G A$ & $73(73 / 348,20.9)^{\star}$ & $19(19 / 44,43.2) \dagger$ & $14(14 / 32,43.8) \dagger$ & $14(14 / 20,70.0)^{\star}$ & $<0.01$ \\
\hline Asphyxia neonatorum & $6(6 / 348,1.7)$ & $2(2 / 44,4.5)$ & $1(1 / 32,3.1)$ & $2(2 / 20,10.0)$ & 0.17 \\
\hline$H D P$ & $31(8.3)^{\star}$ & $21(35.0) \dagger$ & $17(36.9) \dagger$ & $21(61.7)^{\star}$ & $<0.01$ \\
\hline
\end{tabular}

*Significantly different from each other group;

†Significantly different from the $\mathrm{N}$ and $\mathrm{P} 3$ groups;

¥Significantly different from the P2 and P3 groups;

HDP, hypertensive disorders of pregnancy; SGA, small for gestational age.

with SLE. It should be noted that for patients with SLE, multiple pregnancies caused by assisted reproductive technology should be avoided as much as possible.

In addition, our results showed that aspirin use is a protective factor for foetal loss and preterm birth, which is also consistent with other studies. ${ }^{24}{ }^{36}$ The improved pregnancy outcome in SLE pregnancies treated with aspirin appears to correlate with the mechanism of inhibiting platelet aggregation and anti-inflammatory activity, promoting normal uterine artery flow velocity. ${ }^{37}$

A total of eleven cases of asphyxia neonatorum (2.1\%) were recorded in this study. In non-SLE pregnant women, hypertension increases the possibility of placental dysfunction, leading to foetal hypoxia and asphyxia after birth. ${ }^{38}$ The same association for neonatal asphyxia in SLE pregnancy was found in our research.

Early studies have reported that specific predictors of HDP, especially PE complicated by SLE, include aPL positivity, thrombocytopaenia, hypocomplementaemia, disease flares and renal damage. ${ }^{22}{ }^{39-43}$ Although our results are basically consistent with those of previous studies, it is unexpected that aPL positivity and hypocomplementaemia are not independent risk factors for HDP with SLE. Our data indicate that prepregnancy hypertension, renal disorders and thrombocytopaenia are more significant in predicting HDP.

Many studies have only focused on whether ds-DNA or complements are abnormal as predictors of SLE pregnancy outcomes. To clarify the degree of abnormality of these indicators that threaten SLE pregnancy outcomes, we analysed the correlation between ds-DNA, complements and APOs. We found that the value of ds-DNA correlated slightly positively with the incidence of foetal loss. In addition, we found that the incidences of composite foetal APOs and foetal loss in any interval below the lower normal limit, whether complement C3 or $\mathrm{C} 4$, increased with the decrease in complements. These results may explain the clinical phenomenon that some patients with highly elevated ds-DNA did not have APOs, indicating that $\mathrm{C} 3 / \mathrm{C} 4$ could be used as a disease severity scale rather than ds-DNA.

The diagnostic criterion for proteinuria in lupusrelated renal damage is $>0.5 \mathrm{~g} / \mathrm{d}$, while daily protein levels in pregnant women $>0.3 \mathrm{~g}$ at any time during gestation is considered abnormal. ${ }^{44}$ It was proposed that the rate of foetal loss in SLE pregnancy increases significantly when urine protein $>0.5 \mathrm{~g} /$ day. ${ }^{45}{ }^{46}$ In addition, Moroni et al reported that the odds of preterm delivery increase by $15 \%$ for each quarterly increase in proteinuria by $1 \mathrm{~g}$ per day. ${ }^{47}$ However, few studies have shown the effect of proteinuria with a quantity of less than $0.5 \mathrm{~g} / 24$ hours or higher on SLE pregnancy outcomes. In our study, loss of 24-hour urine protein influenced the incidence of APOs. SLE pregnancies without proteinuria before 20 weeks of gestation showed the lowest incidences of foetal APOs and HDPs. Our data indicate that proteinuria $(\geq 0.3 \mathrm{~g} /$ day $)$ in the first 20 weeks of pregnancy can significantly increase the risk of foetal loss, and the premature birth rate was significantly increased when 24-hour urine protein was $>0.5 \mathrm{~g}$. Furthermore, the probabilities of HDP and SGA increased significantly when 24-hour urine protein was greater than $1 \mathrm{~g}$, suggesting that different degrees of urine protein loss correspond to rates of different adverse outcomes in SLE pregnancy. Thus, we found that proteinuria before the $20^{\text {th }}$ gestational week may be more likely to progress to HDP, similar to previous studies not focusing on the SLE population. ${ }^{48}{ }^{49}$ Our data support the hypothesis that dividing 24-hour urine protein values during SLE pregnancy into $0.3 \mathrm{~g}, 0.5 \mathrm{~g}$ and $1 \mathrm{~g}$ can help to predict different APOs. 
The findings in this study contribute to a better counselling and tailoring of obstetric surveillance in SLE pregnancy. Nevertheless, our study had some limitations. As a chart review study, inherent information bias was present. Meanwhile, there is a lack of information on uterine contraction inhibitors and follow-up frequency, which may also have an impact on pregnancy outcome. As a single-centre clinical study, it may lack external validity but also avoids the inconsistency and incomparability of data inherent in multi-centre research. Additionally, despite our large total sample size, larger sample sizes are needed to evaluate the identified predictors.

Overall, established risk factors for each APO were carefully assessed in this study. Indicators with more predictive significance have been screened out from conventional indicators, which may help clinicians predict the pregnancy outcome of patients with SLE more accurately and use more intensive monitoring approaches in SLE pregnancies to minimise the incidence of APOs.

\section{Author affiliations}

${ }^{1}$ Department of Obstetrics and Gynecology, Ren Ji Hospital, School of Medicine, Shanghai Jiao Tong University, Shanghai, China

${ }^{2}$ Shanghai Key Laboratory of Gynecologic Oncology, Shanghai, China

${ }^{3}$ Department of Rheumatology, Ren Ji Hospital, School of Medicine, Shanghai Jiao Tong University, Shanghai, China

${ }^{4}$ Shanghai Institute of Rheumatology, Shanghai, China

${ }^{5}$ State Key Laboratory of Oncogenes and Related Genes, Shanghai Cancer Institute, Ren Ji Hospital, School of Medicine, Shanghai Jiao Tong University, Shanghai, China

Contributors Guarantor: JW and WD. Conception and design: MJ, JW and WD. Administrative support: JW and WD. Provision of study materials or patients: $\mathrm{MJ}$, YC, YW, QF, JW and WD. Collection and assembly of data: MJ, YC, SL, JW, WD. Data analysis and interpretation: MJ, YC, YW, SL, JW, WD. Manuscript writing: all authors. Final approval of manuscript: all authors.

Funding This work was supported by the National Natural Science Foundation of China (Grant no. 81901494 to JY W) and by grants from the Shanghai Municipal Health Commission (Grant no. ZHYY-ZXYJHZX-202019 and GWV-10.2-YQ12 to JY W) and by grants from Shanghai "Rising Stars of Medical Talent" Youth Development Program (SHWSRS $<2021>99$ to JY W) and by grants from the Science and Technology Commission of Shanghai Municipality (Grant no. 18441904800 to W Di).

Competing interests None declared.

Patient consent for publication Not applicable.

Ethics approval The authors are accountable for all aspects of the work in ensuring that questions related to the accuracy or integrity of any part of the work are appropriately investigated and resolved. The study was conducted in accordance with the Declaration of Helsinki (as revised in 2013). The study was approved by the ethics committee of Ren Ji Hospital, Shanghai Jiao Tong University School of Medicine (2017-113). Due to the retrospective nature of the study, informed consent was not required.

Provenance and peer review Not commissioned; externally peer reviewed.

Data availability statement No data are available.

Supplemental material This content has been supplied by the author(s). It has not been vetted by BMJ Publishing Group Limited (BMJ) and may not have been peer-reviewed. Any opinions or recommendations discussed are solely those of the author(s) and are not endorsed by BMJ. BMJ disclaims all liability and responsibility arising from any reliance placed on the content. Where the content includes any translated material, BMJ does not warrant the accuracy and reliability of the translations (including but not limited to local regulations, clinical guidelines, terminology, drug names and drug dosages), and is not responsible for any error and/or omissions arising from translation and adaptation or otherwise.
Open access This is an open access article distributed in accordance with the Creative Commons Attribution Non Commercial (CC BY-NC 4.0) license, which permits others to distribute, remix, adapt, build upon this work non-commercially, and license their derivative works on different terms, provided the original work is properly cited, appropriate credit is given, any changes made indicated, and the use is non-commercial. See: http://creativecommons.org/licenses/by-nc/4.0/.

ORCID iDs

Qiong Fu http://orcid.org/0000-0001-5873-6422

Jiayue Wu http://orcid.org/0000-0002-6247-9489

\section{REFERENCES}

1 Wang Z, Wang Y, Zhu R, et al. Long-Term survival and death causes of systemic lupus erythematosus in China: a systemic review of observational studies. Medicine 2015;94:e794.

2 Li M, Zhang W, Leng X, et al. Chinese SLE treatment and research Group (CSTAR) registry: I. major clinical characteristics of Chinese patients with systemic lupus erythematosus. Lupus 2013;22:1192-9.

3 Ko HS, Ahn HY, Jang DG, et al. Pregnancy outcomes and appropriate timing of pregnancy in 183 pregnancies in Korean patients with SLE. Int J Med Sci 2011;8:577-83.

4 Whitelaw DA, Hall D, Kotze T. Pregnancy in systemic lupus erythematosus: a retrospective study from a developing community. Clin Rheumatol 2008;27:577-80.

5 Phadungkiatwattana P, Sirivatanapa P, Tongsong T. Outcomes of pregnancies complicated by systemic lupus erythematosus (SLE). $J$ Med Assoc Thai 2007;90:1981-5.

6 Buyon JP, Kim MY, Guerra MM, et al. Predictors of pregnancy outcomes in patients with lupus: a cohort study. Ann Intern Med 2015;163:153-63.

7 Bramham K, Hunt BJ, Bewley S, et al. Pregnancy outcomes in systemic lupus erythematosus with and without previous nephritis. $J$ Rheumatol 2011;38:1906-13.

8 Fischer-Betz R, Specker C. Pregnancy in systemic lupus erythematosus and antiphospholipid syndrome. Best Pract Res Clin Rheumatol 2017;31:397-414.

9 Borella E, Lojacono A, Gatto M, et al. Predictors of maternal and fetal complications in SLE patients: a prospective study. Immunol Res 2014;60:170-6.

10 Yelnik CM, Laskin CA, Porter TF, et al. Lupus anticoagulant is the main predictor of adverse pregnancy outcomes in aPL-positive patients: validation of PROMISSE study results. Lupus Sci Med 2016;3:e000131.

11 Martínez-Sánchez N, Pérez-Pinto S, Robles-Marhuenda Ángel, et al. Obstetric and perinatal outcome in anti-Ro/SSA-positive pregnant women: a prospective cohort study. Immunol Res 2017;65:487-94.

12 Palma Dos Reis CR, Cardoso G, Carvalho C, et al. Prediction of adverse pregnancy outcomes in women with systemic lupus erythematosus. Clin Rev Allergy Immunol 2020;59:287-294.

13 Chakravarty EF, Colón I, Langen ES, et al. Factors that predict prematurity and preeclampsia in pregnancies that are complicated by systemic lupus erythematosus. Am J Obstet Gynecol 2005;192:1897-904.

14 Hochberg MC. Updating the American College of rheumatology revised criteria for the classification of systemic lupus erythematosus. Arthritis Rheum 1997;40:1725.

$15 \mathrm{Wu}$ J, Zhang W-H, Ma J, et al. Prediction of fetal loss in Chinese pregnant patients with systemic lupus erythematosus: a retrospective cohort study. BMJ Open 2019;9:e023849.

16 Frey HA, Klebanoff MA. The epidemiology, etiology, and costs of preterm birth. Semin Fetal Neonatal Med 2016;21:68-73.

17 Ludvigsson JF, Lu D, Hammarström L, et al. Small for gestational age and risk of childhood mortality: a Swedish population study. PLoS Med 2018:15:e1002717.

18 Berglund S, Norman M, Grunewald C, et al. Neonatal resuscitation after severe asphyxia--a critical evaluation of 177 Swedish cases. Acta Paediatr 2008:97:714-9.

19 Sutton ALM, Harper LM, Tita ATN. Hypertensive disorders in pregnancy. Obstet Gynecol Clin North Am 2018;45:333-47.

20 Ruperto N, Hanrahan LM, Alarcón GS, et al. International consensus for a definition of disease flare in lupus. Lupus 2011;20:453-62.

21 Piccoli GB, Cabiddu G, Attini R, et al. Risk of adverse pregnancy outcomes in women with CKD. J Am Soc Nephrol 2015;26:2011-22.

22 Chen D, Lao M, Zhang J, et al. Fetal and maternal outcomes of planned pregnancy in patients with systemic lupus erythematosus: a retrospective multicenter study. J Immunol Res 2018;2018:1-7.

23 Clowse MEB. Lupus activity in pregnancy. Rheum Dis Clin North Am 2007;33:237-52. 
24 Kalok A, Abdul Cader R, Indirayani I, et al. Pregnancy outcomes in systemic lupus erythematosus (SLE) women. Horm Mol Biol Clin Investig 2019;40:e31553696.

25 Barnabe C, Faris PD, Quan H. Canadian pregnancy outcomes in rheumatoid arthritis and systemic lupus erythematosus. Int $J$ Rheumatol 2011;2011:1-6.

26 Baer AN, Witter FR, Petri M. Lupus and pregnancy. Obstet Gynecol Surv 2011;66:639-53.

$27 \mathrm{Kim}$ S-Y, Lee J-H. Prognosis of neonates in pregnant women with systemic lupus erythematosus. Yonsei Med J 2008;49:515.

28 Zhan Z, Yang Y, Zhan Y, et al. Fetal outcomes and associated factors of adverse outcomes of pregnancy in southern Chinese women with systemic lupus erythematosus. PLoS One 2017;12:e0176457.

29 Tian X, Li M, Ye Z, et al. Related factors of fetal loss in Chinese women with systemic lupus erythematosus: data from Chinese SLE treatment and research Group registry IV. Int J Rheum Dis 2015;18:654-60.

30 Skorpen CG, Lydersen S, Gilboe I-M, et al. Influence of disease activity and medications on offspring birth weight, pre-eclampsia and preterm birth in systemic lupus erythematosus: a population-based study. Ann Rheum Dis 2018;77:264-9.

31 Jara LJ, Medina G, Cruz-Dominguez P, et al. Risk factors of systemic lupus erythematosus flares during pregnancy. Immunol Res 2014;60:184-92.

32 Eudy AM, Jayasundara M, Haroun T, et al. Reasons for cesarean and medically indicated deliveries in pregnancies in women with systemic lupus erythematosus. Lupus 2018;27:351-6.

33 Liu Q, Yang H, Sun X, et al. Risk factors and complications of small for gestational age. Pak J Med Sci 2019;35:1199-203.

34 Liu J, Zhao Y, Song Y, et al. Pregnancy in women with systemic lupus erythematosus: a retrospective study of 111 pregnancies in Chinese women. J Matern Fetal Neonatal Med 2012;25:261-6.

35 Alami Z, Agier MS, Ahid S, et al. Pregnancy outcome following in utero exposure to azathioprine: a French comparative observational study. Therapie 2018;73:199-207.

36 Salazar-Páramo M, Jara LJ, Ramos A, et al. Longitudinal study of antinuclear and anticardiolipin antibodies in pregnant women with systemic lupus erythematosus and antiphospholipid syndrome. Rheumatol Int 2002;22:142-7.

37 Weiner Z, Lorber M, Blumenfeld Z. Umbilical and uterine artery flow velocity waveforms in pregnant women with systemic lupus erythematosus treated with aspirin and glucocorticosteroids. Am J Reprod Immunol 1992;28:168-71.

38 Arbeille P, Perrotin F, Salihagic A, et al. Fetal Doppler hypoxic index for the prediction of abnormal fetal heart rate at delivery in chronic fetal distress. Eur J Obstet Gynecol Reprod Biol 2005;121:171-7.

39 Wu J, Ma J, Bao C, et al. Pregnancy outcomes among Chinese women with and without systemic lupus erythematosus: a retrospective cohort study. BMJ Open 2018;8:e020909.

40 Larosa M, Del Ross T, Calligaro A, et al. Clinical outcomes and predictors of maternal and fetal complications in pregnancies of patients with systemic lupus erythematosus. Expert Rev Clin Immunol 2019;15:617-27.

41 Lateef A, Petri M. Systemic lupus erythematosus and pregnancy. Rheum Dis Clin North Am 2017;43:215-26.

42 Mecacci F, Simeone S, Cirami CL, et al. Preeclampsia in pregnancies complicated by systemic lupus erythematosus (SLE) nephritis: prophylactic treatment with multidisciplinary approach are important keys to prevent adverse obstetric outcomes. J Matern Fetal Neonatal Med 2019;32:1292-8.

43 Dong Y, Yuan F, Dai Z, et al. Preeclampsia in systemic lupus erythematosus pregnancy: a systematic review and meta-analysis. Clin Rheumatol 2020;39:319-25.

44 Alshohaib S. Outcome of pregnancy in patients with inactive systemic lupus erythromatosus and minimal proteinuria. Saudi J Kidney Dis Transpl 2009;20:802-5.

45 Wagner SJ, Craici I, Reed D, et al. Maternal and foetal outcomes in pregnant patients with active lupus nephritis. Lupus 2009;18:342-7.

46 Moroni G, Quaglini S, Banfi G, et al. Pregnancy in lupus nephritis. Am $J$ Kidney Dis 2002;40:713-20.

47 Moroni G, Doria A, Giglio E, et al. Fetal outcome and recommendations of pregnancies in lupus nephritis in the 21 st century. A prospective multicenter study. J Autoimmun 2016;74:6-12.

48 Morikawa M, Yamada T, Yamada T, et al. Pregnancy outcome of women who developed proteinuria in the absence of hypertension after mid-gestation. J Perinat Med 2008;36:419-24.

49 Chung WH, To WWK. Outcome of pregnancy with new onset proteinuria and progression to pre-eclampsia: a retrospective analysis. Pregnancy Hypertens 2018;12:174-7. 